\title{
Deoxydichlorination of aldehydes catalyzed by Diphenyl sulfoxide
}

\author{
I.A. Gorbunova ${ }^{\text {a }}$, D.A. Shipilovskikh ${ }^{\text {ab }}$, S.A. Shipilovskikh ${ }^{\text {ac* }}$ (i) \\ a: Perm State University, 61499o, Perm, Russia \\ b: Perm National Research Polytechnic University, 614990, Perm, Russia \\ c: ITMO University, 197101, Saint Petersburg, Russia \\ * Corresponding author: s.shipilovskikh@metalab.ifmo.ru \\ This short communication (letter) belongs to the MOSM2021 Special Issue. \\ (c) 2021, The Authors. This article is published in open access form under the terms and conditions of the Creative \\ Commons Attribution (CC BY) license (http://creativecommons.org/licenses/by/4.o/).
}

\begin{abstract}
The diphenyl sulfoxide-catalyzed conversion of aldehydes to 1,1-dichlorides is reported. The reaction proceeds via a sulfurous (IV)-catalysis manifold in which diphenyl sulfoxide turnover is achieved using oxalyl chloride as a consumable reagent.
\end{abstract}

\author{
Keywords \\ aldehydes \\ Lewis base catalysis \\ organocatalysis \\ diphenyl sulfoxide \\ Received: 31.10 .2021 \\ Revised: 17.11.2021 \\ Accepted: 01.12.2021 \\ Available online: 03.12.2021
}

\section{Introduction}

Nucleophilic substitutions $S_{\mathrm{N}}$ are general chemical transformations, as they allow, for example, strategic building of $\mathrm{C}-\mathrm{Cl}, \mathrm{C}-\mathrm{O}, \mathrm{C}-\mathrm{N}$ and $\mathrm{C}-\mathrm{C}$ bonds [1-10]. In addition, geminal dihalides, especially dichlorides, are important intermediates in chemical synthesis, and the traditional synthesis protocols are often limited in terms of cost efficiency and waste balance $[11,12]$. However, research in this area is at an early stage in the study of such catalytic reaction. Although by now several effective protocols for the preparation of dichlorides from aldehydes catalyzed by a Lewis base have been disclosed [13, 14], all possibilities for studying these reactions have not yet been realized (Scheme 1).

Dichlorides - important class of intermediates in organic synthesis. They were used for alkenylation of carbonyl compounds $[15,16]$, cyclopropanation and epoxidation [17-19], dimerization [20, 21] and others [22-25]. In addition, geminal dichlorides are encountered as structural motifs in polyhalogenated natural products [26, 27] (Fig. 1).

\section{Experimental}

Yields are given for isolated products showing one spot on a TLC plate and no impurities detectable in the NMR spectrum. The identity of the products prepared by different methods was checked by comparison of their NMR spectra.
${ }^{1} \mathrm{H}$ and ${ }^{13} \mathrm{C}$ NMR spectra were recorded at $400 \mathrm{MHz}$ for ${ }^{1} \mathrm{H}$ and $100 \mathrm{MHz}$ for ${ }^{13} \mathrm{C} \mathrm{NMR}$ at room temperature; the chemical shifts $(\delta)$ were measured in ppm with respect to the solvent $\left(\mathrm{CDCl}_{3},{ }^{1} \mathrm{H}: \delta=7.26 \mathrm{ppm},{ }^{13} \mathrm{C}: \delta=77.16 \mathrm{ppm}\right.$; [D6] DMSO, $\left.{ }^{1} \mathrm{H}: \delta=2.50 \mathrm{ppm},{ }^{13} \mathrm{C}: \delta=39.52 \mathrm{ppm}\right)$. Coupling constants $(J$ ) are given in Hertz. Splitting patterns of apparent multiplets associated with an averaged coupling constants were designated as $s$ (singlet), $d$ (doublet), $t$ (triplet), $q$ (quartet), sept (septet), $m$ (multiplet), $d d$ (doublet of doublets) and $b r$ (broadened). Melting points were determined with a "Stuart SMP 30", the values are uncorrected. Flash chromatography was performed on silica gel Macherey Nagel (40-63 $\mu \mathrm{m})$.

Reaction progress was monitored by GC/MS analysis and thin layer chromatography (TLC) on aluminum backed plates with Merck Kiesel 6o F254 silica gel. The TLC plates were visualized either by UV radiation at a wavelength of $254 \mathrm{~nm}$, or stained by exposure to a Dragendorff's reagent or potassium permanganate aqueous solution. All the reactions were carried out using dried and freshly distilled solvent.

\subsection{General method for synthesis of dichlorides from aldehyde}

Diphenyl sulfoxide $\left(\mathrm{Ph}_{2} \mathrm{SO}\right)$ ( $40 \mathrm{mg}$, $0.2 \mathrm{mmol}$, 0.1 equiv, $10 \mathrm{~mol} . \%)$ and aldehyde 1 ( $2 \mathrm{mmol}, 1$ equiv) were dissolved in $15 \mathrm{~mL}$ of anhydrous toluene in a $25 \mathrm{~mL}$ round bottom flask equipped with a magnetic stirring bar. The resulting solution was treated dropwise with neat oxalyl chloride (0.26 mL, $3 \mathrm{mmol}, 1.5$ equiv (chlorine source)) 
using an adjustable volume pipette (0.1-1.0 $\mathrm{mL}$ ), followed by the temperature increase up to $100{ }^{\circ} \mathrm{C}$; the mixture was stirred for $6 \mathrm{~h}$. The reaction progress was monitored by GC-MS. After the reaction was complete, the solution was filtered and concentrated in vacuum. The crude mixture thus obtained was purified by flash chromatography on silica (petroleum ether/ $\mathrm{Et}_{2} \mathrm{O}-19 / 1$ ).

\subsection{1. (Dichloromethyl)benzene $4 a$}

Obtained from 1a (212 mg, $2 \mathrm{mmol}$ ), diphenyl sulfoxide $\left(\mathrm{Ph}_{2} \mathrm{SO}\right.$ ) (40 mg, $0.2 \mathrm{mmol}$, 0.1 equiv, $10 \mathrm{~mol} \%$ ), and oxalyl chloride (0.26 mL, $3 \mathrm{mmol}, 1.5$ equiv), in anhydrous toluene $(15 \mathrm{~mL})$. Colorless oil (242 mg, 75\%). ${ }^{1} \mathrm{H}$ NMR $\left(\mathrm{CDCl}_{3}, 400 \mathrm{MHz}\right) \delta(\mathrm{ppm}): 6.75(\mathrm{~s}, 1 \mathrm{H}, \mathrm{CH}), 7.44(\mathrm{~m}, 3 \mathrm{H}$, $\left.\mathrm{H}_{\mathrm{Ar}}\right), 7.66\left(\mathrm{~m}, 2 \mathrm{H}, \mathrm{H}_{\mathrm{Ar}}\right) .{ }^{13} \mathrm{C} \mathrm{NMR}\left(\mathrm{CDCl}_{3}, 100 \mathrm{MHz}\right)$ $\delta$ (ppm): 72.0, 126.2, 128.8, 123.0, 140.4.

\subsubsection{1-(Dichloromethyl)-4-methylbenzene $4 \mathrm{~b}$}

Obtained from $1 \mathrm{~b}$ (240 $\mathrm{mg}, 2 \mathrm{mmol}$ ), diphenyl sulfoxide ( $\mathrm{Ph}_{2} \mathrm{SO}$ ) (40 mg, $0.2 \mathrm{mmol}$, 0.1 equiv, $10 \mathrm{~mol} \%$ ), and oxalyl chloride ( $0.26 \mathrm{~mL}, 3 \mathrm{mmol}, 1.5$ equiv), in anhydrous toluene (15 mL). Colorless oil (278 mg, 80\%). ${ }^{1} \mathrm{H}$ NMR $\left(\mathrm{CDCl}_{3}, 400 \mathrm{MHz}\right) \delta(\mathrm{ppm}): 2.40\left(\mathrm{~s}, 3 \mathrm{H}, \mathrm{CH}_{3}\right), 6.68(\mathrm{~s}, 1 \mathrm{H}$, $\mathrm{CH}), 7.23\left(\mathrm{~m}, 2 \mathrm{H}, \mathrm{H}_{\mathrm{Ar}}\right), 7.48\left(\mathrm{~m}, 2 \mathrm{H}, \mathrm{H}_{\mathrm{Ar}}\right) .{ }^{13} \mathrm{C} \mathrm{NMR}\left(\mathrm{CDCl}_{3}\right.$, $100 \mathrm{MHz}) \delta$ (ppm): 21.7, 71.6, 126.1, 129.3, 137.5, 140.9.

\subsubsection{1-Bromo-4-(dichloromethyl)benzene 4c}

Obtained from 1c (370 $\mathrm{mg}, 2 \mathrm{mmol}$ ), diphenyl sulfoxide ( $\mathrm{Ph}_{2} \mathrm{SO}$ ) (40 mg, $0.2 \mathrm{mmol}$, 0.1 equiv, $10 \mathrm{~mol} \%$ ), and oxalyl chloride ( $0.26 \mathrm{~mL}, 3 \mathrm{mmol}, 1.5$ equiv), in anhydrous toluene (15 mL). Colorless oil (345 mg, 72\%). ${ }^{1} \mathrm{H}$ NMR $\left(\mathrm{CDCl}_{3}, 400 \mathrm{MHz}\right) \delta(\mathrm{ppm}): 6.68(\mathrm{~s}, 1 \mathrm{H}, \mathrm{CH}), 7.46(\mathrm{~m}, 2 \mathrm{H}$, $\left.\mathrm{H}_{\mathrm{Ar}}\right), 7.55\left(\mathrm{~m}, 2 \mathrm{H}, \mathrm{H}_{\mathrm{Ar}}\right) .{ }^{13} \mathrm{C} \mathrm{NMR}\left(\mathrm{CDCl}_{3}, 100 \mathrm{MHz}\right)$ $\delta(\mathrm{ppm}):$ 72.0, 124.3, 128.0, 131.9, 139.4.

\subsubsection{1-(Dichloromethyl)-4-nitrobenzene $4 \mathrm{~d}$}

Obtained from 1d (302 mg, $2 \mathrm{mmol}$ ), diphenyl sulfoxide $\left(\mathrm{Ph}_{2} \mathrm{SO}\right.$ ) (40 mg, $0.2 \mathrm{mmol}$, 0.1 equiv, $10 \mathrm{~mol} \%$ ), and oxalyl chloride (0.26 mL, $3 \mathrm{mmol}, 1.5$ equiv), in anhydrous toluene (15 mL). Colorless oil (259 mg, 63\%). ${ }^{1} \mathrm{H}$ NMR
$\left(\mathrm{CDCl}_{3}, 400 \mathrm{MHz}\right) \delta(\mathrm{ppm}): 6.78(\mathrm{~s}, 1 \mathrm{H}, \mathrm{CH}), 7.78(\mathrm{~m}, 2 \mathrm{H}$, $\left.\mathrm{H}_{\mathrm{Ar}}\right), 8.29\left(\mathrm{~m}, 2 \mathrm{H}, \mathrm{H}_{\mathrm{Ar}}\right) .{ }^{13} \mathrm{C} \mathrm{NMR}\left(\mathrm{CDCl}_{3}, 100 \mathrm{MHz}\right)$ $\delta$ (ppm): 70.2, 124.5, 127.9, 146.6, 149.2.

\subsection{5. (E)-(3,3-Dichloroprop-1-en-1-yl)benzene $4 \mathrm{e}$}

Obtained from 1e (264 mg, $2 \mathrm{mmol}$ ), diphenyl sulfoxide $\left(\mathrm{Ph}_{2} \mathrm{SO}\right.$ ) (40 mg, $0.2 \mathrm{mmol}$, 0.1 equiv, $10 \mathrm{~mol} \%$ ), and oxalyl chloride (0.26 mL, $3 \mathrm{mmol}, 1.5$ equiv), in anhydrous toluene $(15 \mathrm{~mL})$. Colorless oil (286 mg, 77\%). ${ }^{1} \mathrm{H}$ NMR $\left(\mathrm{CDCl}_{3}, 400 \mathrm{MHz}\right) \delta(\mathrm{ppm}): 6.34(d, J=7.6 \mathrm{~Hz}, 1 \mathrm{H}, \mathrm{CH})$, $6.39(d d, J=14.7$ and $7.6 \mathrm{~Hz}, 1 \mathrm{H}, \mathrm{CH}), 6.72(d, J=14.7 \mathrm{~Hz}$, $1 \mathrm{H}, \mathrm{CH}), 7.41(\mathrm{~m}, 5 \mathrm{H}, \mathrm{H} \mathrm{Ar}) .{ }^{13} \mathrm{C} \mathrm{NMR}\left(\mathrm{CDCl}_{3}, 100 \mathrm{MHz}\right)$ $\delta(\mathrm{ppm}): 73.5,127.0,128.2,129.1,129.3,132.5,134.9$.

Denton 2013

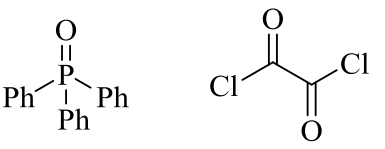

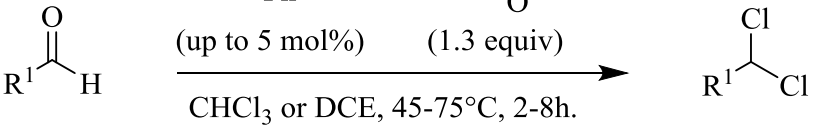

Huy 2019

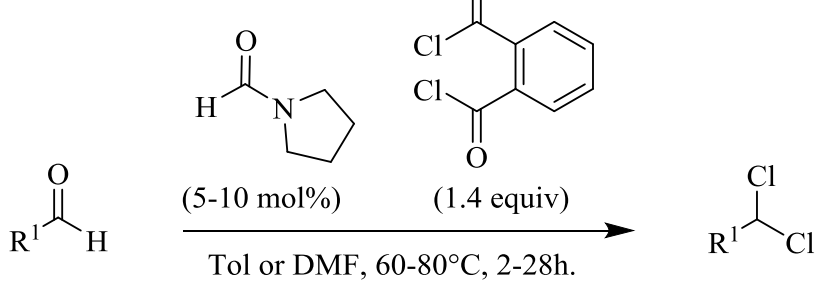

This Work

$$
\begin{aligned}
& \mathrm{Ph}^{-\mathrm{S}_{-}^{\mathrm{O}} \mathrm{Ph}} \mathrm{Cl} \prod_{\mathrm{O}}^{\mathrm{O}} \\
& \mathrm{R}^{1} \stackrel{\mathrm{O}}{\mathrm{C}_{\mathrm{H}}} \underset{(10 \mathrm{~mol} \%) \quad(1.5 \text { equiv })}{\longrightarrow} \mathrm{R}_{\mathrm{Cl}}^{\mathrm{Cl}} \\
& \text { Tol, } 90^{\circ} \mathrm{C}, 6 \mathrm{~h} \text {. }
\end{aligned}
$$

Scheme 1 Deoxydichlorination of aldehydes to 1,1-dichlorides<smiles>O[C@H]1CC[C@@H](O)C1(Cl)Cl</smiles>

Caldariomycin<smiles>O=S(=O)(O)CC(Cl)(Cl)CCCCCCCC[C@H](Cl)C[C@@H](Cl)[C@H](OS(=O)(=O)O)[C@H](Cl)[C@H](Cl)c1ccccc1</smiles>

Danicalipin A

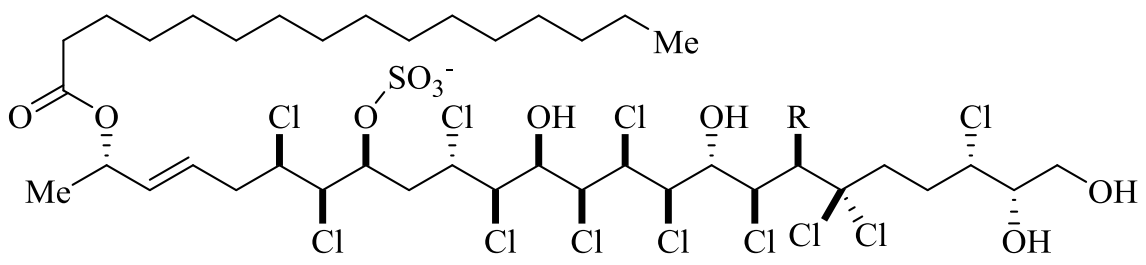

undecachlorosulfolipids $\mathrm{A}(\mathrm{R}=\mathrm{OH})$ and $\mathrm{B}(\mathrm{R}=\mathrm{H})$

Fig. 1 Natural products including a fragment of dichlorides 


\section{Results and discussion}

The investigation commenced with establishing the best conditions for the deoxydichlorination of aldehydes, employing benzaldehyde $1 \mathrm{a}$ as a model substrate (Scheme 2). First, the role of each reagent was evaluated. Oxalyl chloride on its own did not produce (Dichloromethyl)benzene 4 a (Table 1 , entry 1 ). The use of stoichiometric quantities of $\mathrm{Ph}_{2} \mathrm{SO}$ and $(\mathrm{COCl})_{2}$ in acetonitrile resulted in low conversion of $1 \mathrm{a}$ into $4 \mathrm{a}$ (entry 2). With $10 \mathrm{~mol} \% \mathrm{Ph}_{2} \mathrm{SO}$ and 1 equiv of oxalyl chloride, 4 a was formed in $15 \%$ conversion (entry 3 ), which increased to $51 \%$ after change the solvent on toluene (entry 4). The up of the temperature to $100{ }^{\circ} \mathrm{C}$ and use 1.5 equiv of oxalyl chloride to give the best results of conversion to $92 \%$ (entry 11 ).<smiles>O=Cc1ccccc1</smiles>

$1 \mathrm{a}$

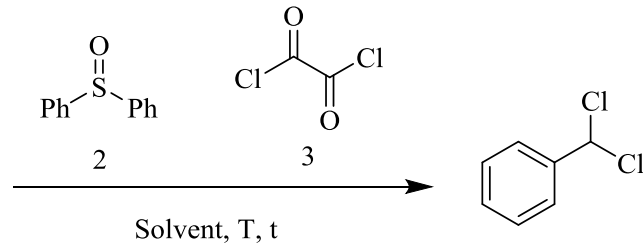

$4 \mathrm{a}$
Scheme 2 The reaction for optimization of the conditions

Table 1 Optimization of the reaction conditions

\begin{tabular}{lcccccc}
\hline Entry & $\begin{array}{c}\text { Equiv of } \\
(\mathrm{COCl})_{2}\end{array}$ & $\begin{array}{c}\mathrm{Ph}_{2} \mathrm{SO}, \\
\mathrm{mol} \%\end{array}$ & Solvent & $\begin{array}{c}T, \\
{ }^{\circ} \mathrm{C}\end{array}$ & $\begin{array}{c}t, \\
\mathrm{~h}\end{array}$ & $\begin{array}{c}\text { Conv., } \\
\%^{\mathrm{b}}\end{array}$ \\
\hline 1 & 1 & - & $\mathrm{MeCN}$ & 50 & 1 & $\mathrm{o}$ \\
\hline 2 & 1 & 100 & $\mathrm{MeCN}$ & 50 & 1 & 19 \\
\hline 4 & 1 & 10 & $\mathrm{MeCN}$ & 50 & 6 & 15 \\
\hline 5 & 1 & 10 & $\mathrm{Tol}$ & 50 & 6 & 53 \\
\hline 6 & 1 & 10 & $\mathrm{DCM}$ & 40 & 6 & 10 \\
\hline 7 & 1 & 10 & $\mathrm{DCE}$ & 50 & 6 & 18 \\
\hline 8 & 1 & 10 & $\mathrm{THF}$ & 50 & 6 & 37 \\
\hline 9 & 1 & 10 & $\mathrm{Et}{ }_{2} \mathrm{O}$ & 30 & 6 & 4 \\
\hline 10 & 1 & 10 & $\mathrm{Tol}$ & 100 & 6 & 85 \\
\hline 11 & 1.5 & 10 & $\mathrm{Tol}$ & 100 & 12 & 88 \\
\hline
\end{tabular}

aGeneral conditions: 1a (0.2 mmol), $\mathrm{Ph}_{2} \mathrm{SO}$, dry solvent $(1 \mathrm{~mL})$, dropwise addition of neat $(\mathrm{COCl})_{2}$. The reactions were carried out for 1-12 h before an aliquot $(50 \mu \mathrm{L})$ was taken, quenched with aqueous solvent ( $1 \mathrm{~mL}$ ), and analyzed by GC.

${ }^{\mathrm{b}}$ Conversion to $4 \mathrm{a}$ was calculated from GC.

The substrate scope was investigated next. As shown in Scheme 3, the reaction work well with different type of aromatic aldehydes, including donor and acceptor substituents at the fourth position of the ring. The use of cinnamaldehyde under the reaction conditions also showed good results.

The proposed mechanism is depicted in Scheme 4. We think that the catalytic cycle start with quick formation of the intermediate chlorodiphenylsulfonium chloride (B) upon treatment of diphenyl sulfoxide (A) with $(\mathrm{COCl})_{2}$. Previously, a similar process was carried out by Denton with triphenylphosphine oxide as a catalyst [14]. Next, in the catalytic cycle, the intermediate B reacts with the aldehyde 1 via oxygen to form the intermediate $\mathrm{C}$, which then undergoes elimination to furnish the geminal dichloride 4 and regenerate the catalyst A.

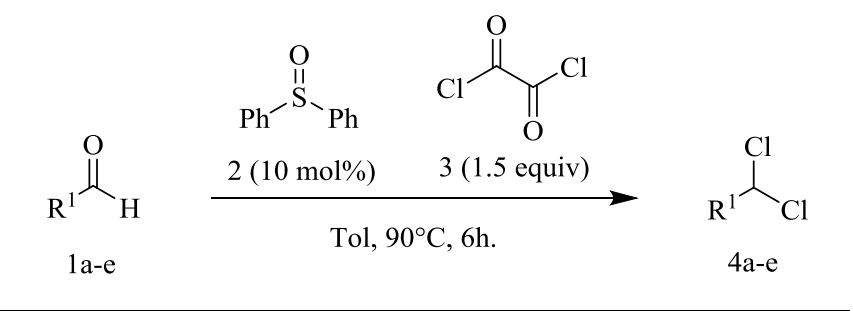<smiles>ClC(Cl)c1ccccc1</smiles>

$4 \mathrm{a}$<smiles>Cc1ccc(C(Cl)Cl)cc1</smiles>

$4 \mathrm{~b}$<smiles>ClC(Cl)c1ccc(Br)cc1</smiles>

$4 \mathrm{c}$<smiles>O=[N+]([O-])c1ccc(C(Cl)Cl)cc1</smiles>

$4 d$<smiles>ClC(Cl)/C=C/c1ccccc1</smiles>

$4 \mathrm{e}$
Scheme 3 Deoxydichlorination of aldehydes catalyzed by Diphenyl sulfoxide

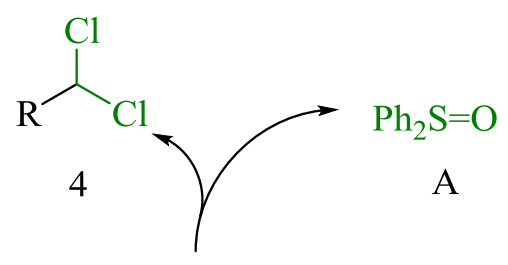

(†)<smiles>[R]C(Cl)(CCl)O[SbH2]</smiles>

$\mathrm{C}$<smiles>[R]C=CC(C)CCC</smiles>

1
Scheme 4 Proposed mechanism

\section{Conclusions}

We have developed a highly expedient protocol for a catalytic deoxydichlorination of aldehydes under conditions of a catalytic Swern Oxidation catalyzed by diphenyl sulfoxide. The salient features of the method are: (i) operational simplicity, (ii) low catalyst loading (10 mol.\%), (iii) medium reaction times and (iv) mild conditions.

\section{Acknowledgments}

The authors thank Russian Science Foundation for grant 20-73-00o81. 


\section{References}

1. Huy PH. Lewis base catalysis promoted nucleophilic substitutions - recent advances and future directions. Eur J Org Chem. 2020;(1):10-27. doi:10.1002/ejoc.201901495

2. Beddoe RH, Sneddon HF, Denton RM. The catalytic Mitsunobu reaction: a critical analysis of the current state-of-the-art. Org Biomol Chem. 2018;16(42):7774-7781. doi:10.1039/C8OB01929K

3. Shipilovskikh SA, Rubtsov AE. Dehydration of oxime to nitriles. AIP Conf Proc. 2019;2063:030019.

doi: $10.1063 / 1.5087327$

4. Huy PH, Hauch T, Filbrich I. Lewis base catalyzed nucleophilic substitutions of alcohols. Synlett. 2016;27(19):26312636. doi:10.1055/s-0036-1588633

5. Kohlmeyer C, Schäfer A, Huy PH, Hilt G. Formamidecatalyzed nucleophilic substitutions: mechanistic insight and rationalization of catalytic. ACS Catal. 2020;10(19):1156711577. doi:10.1021/acscatal.oc03348

6. Shipilovskikh SA, Vaganov VY, Denisova EI, Rubtsov AE, Malkov AV. Dehydration of amides to nitriles under conditions of a catalytic appel reaction. Org Lett. 2018;20(3):728731. doi:10.1021/acs.orglett.7b03862

7. Huy PH, Mbouhom C. Formamide catalyzed activation of carboxylic acids - versatile and cost-efficient amidation and esterification. Chem Sci. 2019;10:7399-7406. doi:10.1039/C9SC02126D

8. Motsch S, Schütz C, Huy PH. Systematic evaluation of sulfoxides as catalysts in nucleophilic substitutions of alcohols. Eur J Org Chem. 2018:4541-4547. doi:10.1002/ejoc.201800907

9. Huy PH, Filbrich I. A general catalytic method for highly costand atom-efficient nucleophilic substitutions. Chem Eur J. 2018;24:7410. doi:10.1002/chem.201800588

10. Fukazawa Y, Vaganov VY, Shipilovskikh SA, Rubtsov AE, Malkov AV. Stereoselective synthesis of atropisomeric bipyridine $\mathrm{N}, \mathrm{N}^{\prime}$-dioxides by oxidative coupling. Org Lett. 2019;21(12):4798-4802. doi:10.1021/acs.orglett.9b01687

11. Takeda T, Endo Y, Reddy ACS, Sasaki R, Fujiwara T. Transformation of ketones into 1-chloro and 1,1-dichloro-1-alkenes by means of a polychloromethane-titanocene(II) system. Tetrahedron. 1999;55:2475. doi:10.1016/S0040-4020(99)00021-6

12. Takeda T, Sasaki R, Fujiwara T. Carbonyl Olefination by Means of a gem-Dichloride- $\mathrm{Cp}_{2} \mathrm{Ti}\left[\mathrm{P}(\mathrm{OEt})_{3}\right]_{2}$ system. J Org Chem. 1998;63(21):7286-7288. doi:10.1021/jog80724h

13. Huy PH. Formamide catalysis facilitates the transformation of aldehydes into geminal dichlorides. Synthesis. 2019;51(12):2474-2483. doi:10.1055/s-0037-1611798

14. An J, Tang X, Moore J, Lewis W, Denton RM. Phosphorus(V)catalyzed dichlorination reactions of aldehydes. Tetrahedron. 2013;69:8769-8776. doi:10.1016/j.tet.2013.07.100
15. Concellón JM, Rodríguez-Solla H, Díaz P, Llavona R. The first sequential reaction promoted by manganese: complete stereoselective synthesis of (E)- $\alpha, \beta$-unsaturated esters from 2,2dichloroesters and aldehydes. J Org Chem. 2007;72:4396. doi:10.1021/joo70209w

16. Concellón JM, Rodríguez-Solla H, de Amo V, Díaz P. Stereoselective olefination reactions promoted by rieke manganese. Synthesis. 2009;15:2634-2645. doi:10.1055/s-0029-1216880

17. Oudeyer S, Leonel E, Paugam JP, Nédélec JY. Formation of epoxides and $\mathrm{N}$-arylaziridines via a simple $\mathrm{Mg}$-Barbier reaction in DMF. Tetrahedron. 2014;70:919-923. doi:10.1016/j.tet.2013.12.016

18. Zhou YY, Uyeda C. Reductive cyclopropanations catalyzed by dinuclear nickel complexes. Angew Chem Int Ed. 2016;55:3171-3175. doi:10.1002/anie.201511271

19. Durán-Peña MJ, Flores-Giubi ME, Botubol-Ares JM, Harwood LM, Collado IG, MacÍas-Sánchez AJ, Hernández-Galán R. Chemoselective and stereoselective lithium carbenoid mediated cyclopropanation of acyclic allylic alcohols. Org Biomol Chem. 2016;14(9):2731-2741. doi:10.1039/c50bo2617b

20. Barrero AF, Herrador MM, Del Moral JFQ, Arteaga P, Akssira M, El Hanbali F, Arteaga JF, Diéguez HR, Sánchez EM. Couplings of benzylic halides mediated by titanocene chloride: Synthesis of bibenzyl derivatives. J Org Chem. 2007;72(6):2251-2254. doi:10.1021/jo062492p

21. Eisch JJ, Qian Y, Rheingold AL. Nickel(II)-carbene intermediates in reactions of geminal dihaloalkanes with nickel(o) reagents and the corresponding carbene capture as the phosphonium ylide. Eur J Inorg Chem. 2007;(11):1576-1584. doi:10.1002/ejic.200601106

22. Giannerini M, Fañanas-Mastral M, Feringa BL. $Z$-Selective Copper-Catalyzed Asymmetric Allylic Alkylation with Grignard Reagents. J Am Chem Soc. 2012;134(9):4108-4111. doi:10.1021/ja300743t

23. Li H, Müller D, Guénée L, Alexakis A. Copper-catalyzed enantioselective synthesis of axially chiral allenes. Org Lett 2012;14(23):5880-5883. doi:10.1021/ol302790e

24. Li H, Grassi D, Guénée L, Bürgi T, Alexakis A. Coppercatalyzed propargylic substitution of dichloro substrates: Enantioselective synthesis of trisubstituted allenes and formation of propargylic quaternary stereogenic centers. Chem Eur J. 2014;20(50):16694-16706. doi:10.1002/chem.201404668

25. Brześkiewicz J, Loska R, Makosza M. $\alpha$-Chlorobenzylation of nitroarenes via vicarious nucleophilic substitution with benzylidene dichloride: Umpolung of the friedel-crafts reaction. J Org Chem. 2018;83(15):8499-8508. doi:10.1021/acs.joc.8b01091

26. Nilewski C, Carreira EM. Recent advances in the total synthesis of chlorosulfolipids. Eur J Org Chem. 2012;(9):1685-1698. doi:10.1002/ejoc.201101525

27. Chung WJ, Vanderwal CD. Stereoselective halogenation in natural product synthesis. Angew Chem Int Ed. 2016;55:4396-4434. 